\title{
Once More, Into the Soup
}

\author{
Pieter Jan Stappers, Delft University of Technology \\ Robert R. Hoffman, Institute for Human and Machine Cognition
}

$\mathbf{I}$ one of the earlier essays in this department, we discussed a number of acronyms all having to do with system design, and all having the form " $x$-centered design." 1 The purpose of that essay was to demonstrate a broad framework within which to understand human-centered computing (HCC), and also to show the various convergences and divergences of the communities of practice that have introduced their own $x$-centered-design designations. Among them are learner-centered design, client-centered design, designer-centered design, decision-centered design, and work-oriented design.

Using a concept map, that essay converged on the things that the $x$-centered-design designations have in common:

- A shared goal: The creation of technologies to enhance humans. This is, of course, what HCC is all about.

- Shared methods (cognitive field research and psychosocial evaluation). This gives HCC a methodology to anchor the creation of intelligent systems in the empirics of cognitive work. ${ }^{2}$

- Shared focus on the high-level or "macrocognitive" processes that define cognitive work, processes such as situation assessment, replanning, problem detection, and decision making. ${ }^{3}$

It comes as little surprise that since the publication of that essay, even more acronyms have been put forth, as individuals and organizations struggle to make their ideas and contributions have an impact. ${ }^{4}$

Our purpose in this follow-up essay is to present an alternative view. To extend the soup metaphor from that earlier essay, what we seek to do here is clarify the broth and pick out the meaty bits. In this alternative view, we don't focus on historical origins of the $x$-centered-design designations in disciplines, or on the counterclaims and clashes of communities of practice. Rather, we take a functional approach, 5,6 in which we don't regard designing monolithically as a single activity. As in the first of the soup essays, we use a diagram.

\section{Design}

The research group at the Industrial DesignStudioLab (ID-StudioLab) of Delft University of Technology studies designers and design activities. It develops new methods, techniques, and tools to support design, including its own design processes. The researchers sometimes found it difficult to express what the ID-StudioLab was all about. In caricature, an unguarded moment could deliver, "We study how designers design in order to design methods to improve design." Responses included: "So, is what you do research, or is it design?" "Are you just designing for yourself?" and "Huh?"

These confusions arise often in discussions in this field. Short words such as "designer," "user," and "research" carry too many common connotations and denotations to be sufficient in themselves. Thus, ID-StudioLab created a diagram to depict the functional process of design and the lab's particular approach. The diagram iterated from a simple one into a larger one, as more and 


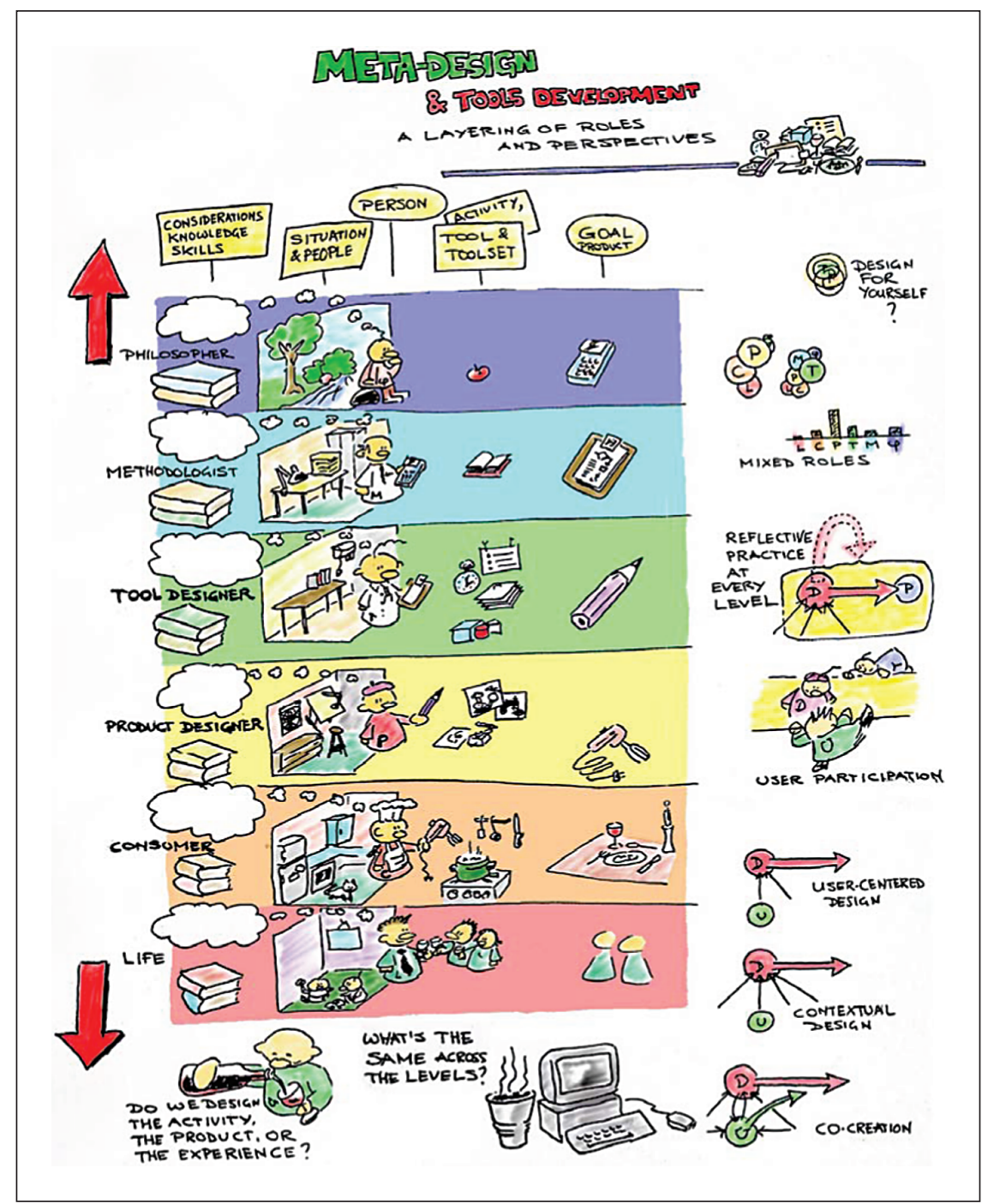

Figure 1. The metadesign diagram, which describes the design of technology as a distributed and collaborative process that involves multiple roles.

more issues and questions seemed to fit neatly into it. The end result is a sketch of the terrain that designers inhabit, a sketch that invites the viewer to look for parallels, similarities, and differences.

Figure 1 presents the final diagram, called the metadesign diagram. (Readers can contact the first author to download a poster-format highresolution version.) The diagram is not a strict or precise graph; it is a sketch to aid thinking about the functional relations among many roles and goals. For example, it was not easy to concretize or symbolize the "product" of the "philosopher." The calculator in that position now is perhaps silly, but a better concept just didn't turn up. The calculator, like the other icons, has the value of being point-at-able, and it serves our purposes largely by its place in the diagram.

\section{Working through the Diagram}

Toward the center of the diagram is the product designer (cartoonishly identified by the beret) who uses tools (exemplified by the pencil, which stands for a variety of tools such as brainstorms, CAD, user studies, and so on) to create consumer products (the mixer).
The design techniques group at the ID-StudioLab deals with developing new "pencils" on the basis of understanding how designers do designing. The tools that the ID-StudioLab uses for this are design methods for conceiving and prototyping, and research methods for studying designing and evaluating the new concept tools. So the product designers are the tool designers' users, in the same way that the consumer who uses the mixer is the product designer's user. This parallel shows why the diagram refers to metadesign-that is, design applied to design.

The diagram is built on two principles: First, it makes sense to use the words "designer" and "user" to refer to roles relative to a specific product. These form a triad of designer makes product for user: <designer, product, user $>$. A common source of confusion in talking about design is that many discussions use the term "designer" to refer to a particular person with a particular professional education, in expressions such as, "designers are not users." Here we take "designer" and "user" to be people's roles with respect to a product or tool.1,6 The diagram distinguishes several roles (such as product designer, tool designer, and consumer); none of these is called just "designer," but each of them plays a designing role with regard to the product to its right in the diagram. The diagram's second principle is that these designer and user roles can be extended and repeated on metalevels; and these "steps" (activities at many levels) can be repeated a few times, while still making sense.

With these basic ideas in mind, let's walk through the levels of the diagram. Starting first at the "product designer," the triad here is <product designer, mixer, consumer $>$. Moving downwards, the level below shows that the consumer is not just a 
passive receiver, but uses the mixer as a tool for preparing a meal (itself a designing activity), which may be used by a host <consumer, meal, host> to entertain his guests <host, party, guests $>$.

We can also work upward. We have already described how the IDStudioLab provides new tools for designers, and this introduces the triad <tool designer, pencil, product designer>. In developing tools for design work, researchers use standard research and design methods, but they have to adapt them as well. This puts researchers in the role of adjusting (redesigning) their methods-for example, determining whether or how to use statistics or modeling and how to evaluate findings. This doesn't stop with using statistics handbooks as dictates and recipes; occasionally researchers make excursions into such fundamental questions as, "What is creativity?" "How exactly do we weigh proof?" and "How do we set the balance between relevance and validity?" Such questions touch upon the metaphysics of thinking. For this designing activity, we reserve a higher level, < philosopher, concepts, methodologist $>$.

The levels are an exercise in ordering thought, but they don't have the strict definition of a house built of bricks. We aren't claiming that there are exactly six levels, or that the separations can always be clearly made. Earlier, we used scare quotes around the word "steps" because all the activities in the diagram are parallel. ${ }^{2}$ But the diagram does help to position some phenomena, call out the parallels, and clarify some misunderstandings that may be due to subtle shifts between levels. The diagram's visual form lets us see and point to elements about which we must be clear. Here are a few things that the levels can express.

\section{Attention to Systems, Context, and Cultures}

In the past decade, there has emerged a "contextualist" consensus that designers don't design objects to be used in a vacuum, or to be used for an isolated technical function. ${ }^{7}$ In designing at the level of meanings for users, designers must take into account the situation in which the product is to be used, including the other tools and products in the domain (a mixer is used in a kitchen, together with a bowl and close to an electrical socket), the cultural values and needs that reign there, and the purposes (toward a lower metalevel, perhaps).

The relation between user and designer has changed over the past decades, in terms of who informs, who creates, and who decides. In usercentered design (UCD), consideration of the user's abilities, needs, and values are key to the definition of the new product. This was a step beyond classic "technology push," in which considerations of user and use were secondary. In the piece of the metadesign diagram reproduced in Figure 2, UCD is depicted as a thin arrow, indicating that the designer takes in information about the user, the task, and other tools involved before and during the time that product creation (thick arrow) takes place. The user's role may be that of either a passive object (observed, for example, with ethnographic techniques) or an active participant (taking part in a focus group, for example, or being interviewed).

With contextual design and context mapping, the designer takes into account a broader scope of factors about the user's environment, again with the user either passive or active. ${ }^{6}$ With co-creation, or participatory design, the user not only informs the designer but closely interacts with the designer (double thin arrows), and participates not just in describing the

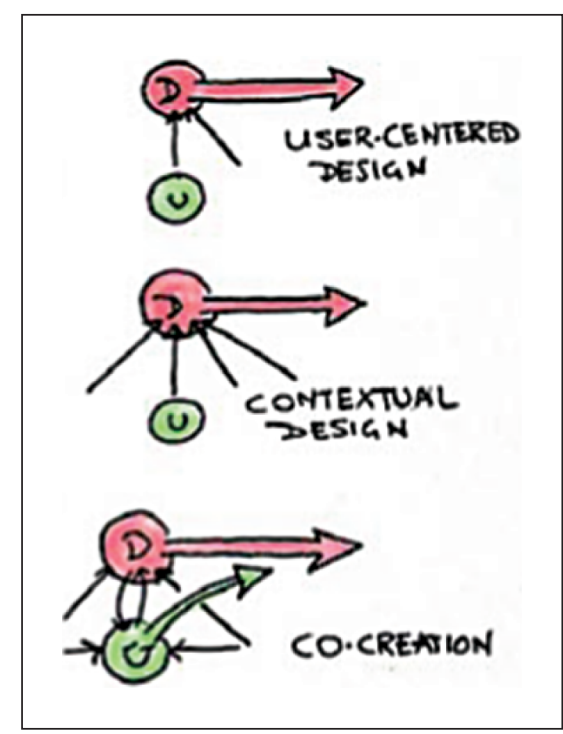

Figure 2. Relationships between design and user. In user-centered design, the designer takes in information about the user, the task, and other tools involved before and during product creation. With contextual design, the designer takes into account a broader scope of factors about the user's environment. In co-creation, the user closely interacts with the designer and participates in generating the new product.

current situation or future needs, but in the actual generation of the new product.

Even close colleagues can differ in their opinions about which players should take which roles, but our jargon does not help us to be explicit about what we mean; being able to mark these roles helps to achieve clarity.

\section{Specific Knowledge}

At each different level there is specific knowledge (symbolized in the metadesign diagram by the books on the left). For instance, one expects the methodologist and academic researchers to come with relevant disciplinary theory, but the consumer's cooking activities also employ specific knowledge.

\section{Values and Culture Differ across Levels}

We've mentioned differences in consumer cultures; the divide between design and research is similar. ${ }^{8}$ 
Researchers and designers typically look for different things in a piece of research; they place different emphases on internal validity (truth) versus external validity (relevance). Distinguishing the levels of metadesign may help to clarify misunderstandings-for example, when two parties in a discussion are missing each other's point because they employ values from different contexts or levels.

\section{Everyone Is a Designer in Some Things}

In the last few years, the idea of "emancipating the user" has received attention, and design researchers, consumer activists, and corporate campaigns (such as Ikea's) have pointed out that everybody has creative abilities. In relation to the metadesign diagram, this would mean that at every level we see people create tools with the purpose of being used or enjoyed by others or themselves. The user-consumer is not just a passive digester of products. But when we focus on a single level, we tend to attribute less creativity and complexity to the levels above and below. Seeing users as passive digesters can be as wrong as seeing methods specialists as uncreative rulebook followers. The metadesign diagram helps us remain aware of that.

\section{Importance of Ethnography to Design}

Ethnography is closely tied to the attention to context mentioned earlier. All the confusion which led us to make the diagram arose when we started to apply ethnographic and contextual techniques in studying the way designers work in design studios. Then we were acting as designers, researchers, and users at the same time. Things became easier when we also inserted these ethnographic techniques in the

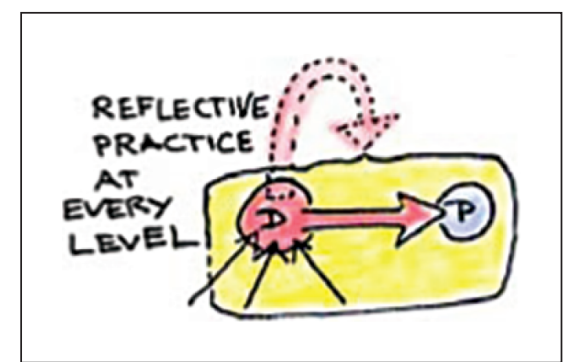

Figure 3. Reflective practice means developing an awareness of the levels besides your own in the metadesign process so that you can work and communicate across levels.

educational program in industrial design, for then the research is aimed at consumers in general, and the results given to designers. In the last few years, the means to study or involve the users intensively in design are becoming popular, both in design education and in industrial practice.

\section{Reflective Practice and Education}

Donald Schön introduced the notion of the reflective practitioner to design theory, indicating that professionals acquire expertise by reflecting on the actions they take. ${ }^{9}$ In design education, the implication is that a design student doesn't just learn the tools and techniques as strict procedures to execute as a computer would, but develops an awareness on a higher level (in the diagram, the tool developer level), so that he can later adapt and refine the methods according to shifting needs of the situation and growing personal skills. The reflective practitioner rises one level higher (Figure 3).

\section{Participatory Design}

Since the 1980s, the field or community of practice of participatory design has gained many advocates. This approach gives the user a more prominent role in the design process. Whereas earlier theories considered users unable to rise above their level of use, the participatory design movement proclaims that by giving users appropriate tools and training, they can become experts in their domain who can contribute on a par with other professionals in the design process. ${ }^{6,10}$ These tools and training serve, as it were, to make them reflective practitioners.

\section{Metadesign and Intelligent Systems}

All of the levels in the metadesign diagram are about people acting, and this gives rise to many similarities across levels. We might mention the computer as a particular similarity: as computer technology has become more broadly intelligent, the computer is the "tool" used by people at all levels. But by reason of this very growth in intelligence capabilities, we don't include the computer in the metadesign diagram because the computer has become the thinking person's ubiquitous tool.

If we use Miller's law (that people can manage $7 \pm 2$ pieces of information in correct serial order in their short-term memory) to teach design students not to overload computer menus with too many items, shouldn't we apply the same principle to the way we design theories, including theories of design? The designers and researchers themselves also have limited mental capabilities, and the theories are their tools. Of course scientists do pay attention to this, calling upon Occam's razor or aesthetic principles to make theories manageable. But it would be worthwhile to train computer scientists involved in creating our design tools to devote more explicit attention to usecentered considerations.

eeing that the "designer" at the different levels is a role-not an individual or a profession-may 


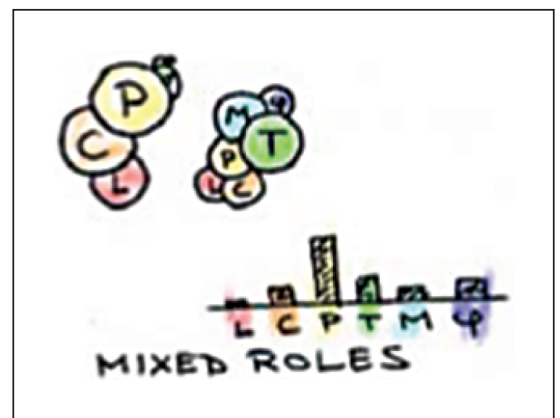

Figure 4. The "designer" at each level of the metadesign process is a role, not an individual or a profession. Most participants in the design process shift among levels and play many roles to varying degrees.

help us realize how most of us shift among the levels (Figure 4). Still, some of us feel most comfortable at one level and spend most of our time there, while others specialize at another level. But each of us should understand the levels, and be able to bridge with others across the levels. We must teach our students to work across and between levels, and we must remember, ourselves, where we and others are.

\section{References}

1. R.R. Hoffman et al., "A Rose by Any Other Name ... Would Probably Be Given an Acronym," IEEE Intelligent Systems, vol. 17, no. 4, 2002, pp. 72-80.

2. G. Klein, "Macrocognition," IEEE Intelligent Systems, vol. 18, no. 3, 2003, pp. 81-85.

3. G. Klein, B. Moon, and R.R. Hoffman, "Making Sense of Sensemaking 1: Alternative Perspectives," IEEE Intelligent Systems, vol. 21, no. 4, 2006, pp. 22-26.

4. R.R. Hoffman, D.O. Norman, and J. Vagners, "Complex Sociotechnical Joint Cognitive Work Systems?” IEEE Intelligent Systems, vol. 24, no. 3, 2009, pp. 82-89.

5. R.R. Hoffman, A. Roesler, and B.M. Moon, "What Is Design in the Context of Human-Centered Computing?" IEEE Intelligent Systems, vol. 19, no. 4, 2004, pp. 89-95.

6. E.B.-N. Sanders and P.J. Stappers, "Co-Creation and the New Landscapes of Design," Codesign, vol. 4, no. 1, 2008, pp. 5-18.
7. H. Beyer and K. Holtzblatt, Contextual Design: Defining Customer-Centered Systems, Academic Press, 1998.

8. E.B.-N. Sanders, "Information, Inspiration and Co-creation," Proc. 6th Int'l Conf. European Academy of Design, 2005; http://www.ead.lancs.ac.uk/conf/ EAD_06.html.

9. D.A. Schön, The Reflective Practitioner: How Professionals Think in Action, Basic Books, 1983.

10. P. Ehn, Work-Oriented Design of Computer Artifacts, Arbetslivscentrum, 1988.

Pieter Jan Stappers is a professor of industrial design engineering at Delft University of Technology, The Netherlands. Contact him at p.j.stappers@tudelft.nl.

Robert R. Hoffman is a senior research scientist at the Institute for Human and Machine Cognition. Contact him at rhoffman@ ihmc.us.

\section{ADVERTISER INFORMATION SEPTEMBER/OCTOBER 2009 • IEEE INTELLIGENT SYSTEMS}

\author{
Advertising Personnel \\ Marion Delaney \\ IEEE Media, Advertising Dir. \\ Phone: +1 4158634717 \\ Email:mdieeemedia@ieee.org \\ Marian Anderson \\ Sr. Advertising Coordinator \\ Phone: +17148218380 \\ Fax: +1 7148214010 \\ Email:manderson@computer.org \\ Sandy Brown \\ Sr. Business Development Mgr. \\ Phone: +17148218380 \\ Fax: +17148214010 \\ Email:sb.ieeemedia@ieee.org
}

\section{Advertising Sales Representatives}

Recruitment:

Mid Atlantic

Lisa Rinaldo

Phone: +1 7327720160

Fax: +1 7327720164

Email:Ir.ieeemedia@

ieee.org

New England

John Restchack

Phone: +1 2124197578

Fax: +1 2124197589

Email:j.restchack@

ieee.org

Southeast

Thomas M. Flynn

Phone: +17706452944

Fax: +1 7709934423

Email:flynntom@

mindspring.com
Midwest/Southwest

Darcy Giovingo

Phone: +1 8474984520

Fax: +1 8474985911

Email:dg.ieeemedia@

ieee.org

Northwest/Southern CA

Tim Matteson

Phone: +1 3108364064

Fax: +1 3108364067

Email:tm.ieeemedia@ieee.org

Japan

Tim Matteson

Phone: +1 3108364064

Fax: +1 3108364067

Email:tm.ieeemedia@ieee.org

\section{Europe}

Hilary Turnbull

Phone: +44 1875825700

Fax: +44 1875825701

Email: impress@

impressmedia.com
Product:

US East

Dawn Becker

Phone: +1 7327720160

Fax: +1 7327720164

Email:db.ieeemedia@ieee.org

US Central

Darcy Giovingo

Phone: +1 8474984520

Fax: +1 8474985911

Email:dg.ieeemedia@ieee.org

US West

Lynne Stickrod

Phone: +1 4159319782

Fax: +1 4159319782

Email: Is.ieeemedia@ieee.org

Europe

Sven Anacker

Phone: +49 2022716911

Fax: +49 2022716920

Email: sanacker@intermediapartners.de 